\title{
Identification of a Novel Regulator for the Escherichia coli fit Iron Trans- port System
}

\author{
Zhiming Ouyang ${ }^{1}$ and Richard Isaacson*
}

\author{
${ }^{I}$ Department of Microbiology, University of Texas Southwestern Medical Center, Dallas, Texas, USA; *Department of \\ Veterinary and Biomedical Science, University of Minnesota, ST PAUL, MN, USA
}

\begin{abstract}
The Escherichia coli fit iron transport system consists of 6 genes, fitA, B, C, D, E and fitR. Based on in silico analysis, FitA-E composes a typical bacterial iron transporter, while FitR was deduced to be a regulator. In this paper the regulation of fit expression by FitR was studied using a quantitative RT-PCR technique and a lacZ reporter assay. It was found that fit expression was repressed when FitR was over-expressed and de-repressed when fitR was knocked out by mutation. When the mutation in fitR was complemented in trans- with the wild type fitR gene, repression of fit expression by FitR was restored. Finally, recombinant FitR was found to bind to the fit promoter DNA when employed in an electrophoretic mobility-shift assay. These results demonstrated that fitR encodes an auto-repressor for the E. coli fit system.
\end{abstract}

\section{INTRODUCTION}

Iron is an essential element for bacterial growth and pathogenesis. Although there is sufficient iron in the environment, iron is not readily available to bacteria in animal or human hosts [1]. To support growth, bacteria have evolved to possess multiple iron transport systems to acquire iron from the environment or hosts. In a previous study, a novel putative $\mathrm{ABC}$ iron transport system, fit, was identified in an E. coli strain causing human septicemia [2]. The E. coli fit system consists of 6 genes designated fitA, $-B,-C,-D,-E$ and fitR, encoding an outer-membrane receptor protein (FitA), a periplasmic binding protein (FitE), two permease proteins (FitC and -D), an ATPase (FitB), and a hypothetical protein (FitR) [2]. Gene fit $A,-B,-C,-D,-E$ encode a typical bacterial iron transporter. Although there is no match for the predicted FitR protein through BLAST analysis, sequence analysis found that an N-terminal helix-turn-helix DNA binding motif and a C-terminal sugar isomerase domain were present in the predicted FitR. This structure is conserved in many bacterial transcriptional regulators, such as E. coli RpiR, LpcA, GutQ, and Glms [3, 4]. In this paper, quantitative RT-PCR and a fit-lacZ reporter analysis were employed to study the expression of fit system under conditions of fitR overexpression, and mutation and complementation of fitR. It was found that fitR repressed fit expression.

\section{METHODS}

\section{Bacterial Strains and Media}

The bacterial strains and plasmids used in this study are listed in Table 1. All strains were grown in Luria-Bertani (LB) media. All reagents and media were made with deion-

*Address correspondence to this author at the Department of Veterinary and Biomedical Science, University of Minnesota, ST PAUL, MN, USA; Tel: (612) 624-0701; Fax: (612)625-5203; E-mail: isaac015@umn.edu

Current Address: Department of Microbiology, University of Texas Southwestern Medical Center, Dallas, Texas, USA. ized water after passage through a Millipore Catridge system (Millipore, USA). All glassware was treated with $8 \mathrm{M} \mathrm{HCl}$ and then rinsed 3 times with double distilled $\mathrm{H}_{2} \mathrm{O}$. When appropriate, supplements were added to media at following concentrations: ampicillin, $100 \mu \mathrm{g} / \mathrm{ml}$; tetracycline, 15 $\mu \mathrm{g} / \mathrm{ml}$; kanamycin, $50 \mu \mathrm{g} / \mathrm{ml}$; trimethoprim, $100 \mu \mathrm{g} / \mathrm{ml}$.

\section{Recombinant DNA Techniques}

General genetic techniques including PCR, genomic or plasmid DNA purification, ligation and transformation were performed as described previously [2]. Restriction endonuclease and DNA-modifying enzymes were used according to the manufacturer's recommendations. DNA fragments were purified from agarose gels using Qiaquick gel extraction kit (Qiagen, USA). DNA cloning and manipulation were conducted in E.coli DH5 $\alpha$ cells. All oligonucleotide primers were commercially synthesized by IDT (Integrated DNA Technologies, USA) and listed in Table 2. DNA sequencing work was done at the Advanced Genetic Analysis Center (AGAC) at the University of Minnesota.

\section{Construction of Transposon-Insertional Mutants}

The EZ::TN transposons (Epicentre, Madison, Wisconsin, USA) were used to create gene disruption mutants in $E$. coli i484 through in vitro transposon insertional mutagenesis according to the protocols provided by the manufacturer [5, 6]. Briefly, the particular gene to be mutated was amplified by PCR. After purification, an EZ::TN transposon was inserted into the DNA fragment through in vitro transposition using the EZ::TN transposase. The disrupted DNA was then electroporated as linear DNA into a recipient strain containing the plasmid pKD46 [5]. Mutants were screened for antibiotic resistance encoded on the inserted EZ::TN transposon and the presence of the transposon in the mutated gene was confirmed by PCR amplification and DNA sequence analysis. Plasmid pKD46 was then eliminated from these cells by growth at $42^{\circ} \mathrm{C}$. 
Table 1. Bacterial Strains and Plasmids Used in this Study

\begin{tabular}{|c|c|c|}
\hline Strain and Plasmid & Deskription & Reference \\
\hline \multicolumn{3}{|l|}{ E. coli strains } \\
\hline oy077 & $\mathrm{i} 484$, fitR $::$ EZ-TN5 $<$ Kan-2 > & This study \\
\hline oy016 & i484, lacZ :: EZ-TN5 < DHFR-1 > & This study \\
\hline oy022 & oy 016 carrying pMP-fitA & This study \\
\hline оу026 & oy016, fitR :: EZ-TN5 < Kan-2> & This study \\
\hline oy028 & oy026 carrying pMP-fitB & This study \\
\hline DH5 $\alpha$ & $\begin{array}{c}F-, \phi 80 d \text { lacZAM15 } \Delta \text { (lacZYA-argF) U169, endA1, recAl, hsdS17 (rK-mK+), deoR, thi-1, } \\
\text { supE44, } \lambda-, \text { gyrA96, relA1 }\end{array}$ & Invitrogen \\
\hline \multicolumn{3}{|l|}{ Plasmids } \\
\hline pMP220 & Contains a promoterless $l a c Z$ gene, Tet $^{\mathrm{r}}$ & 19 \\
\hline pMP-fitA & fit promoter was cloned into pMP220 with a direction of $f i t A$ transcription & 2 \\
\hline pMP-fitB & fit promoter was cloned into pMP220 with a direction of $f i t B$ transcription & 2 \\
\hline
\end{tabular}

\section{RNA Extraction and Real-Time RT-PCR Analysis}

Bacteria were grown in LB with appropriate supplements at $37^{\circ} \mathrm{C}$ with shaking. After 3 hours, cells were harvested, lysed and RNA was isolated using the Qiagen RNeasy Mini Kit (Qiagen, USA). Reverse transcription was performed using the SuperScript ${ }^{\mathrm{TM}}$ III Reverse Transcriptase (Invitrogen, USA). Real-time PCR (qPCR) using Platinum ${ }^{\circledR}$ SYBR ${ }^{\circledR}$ Green qPCR SuperMix-UDG (Invitrogen, USA) was then performed to measure the level of gene expression. The genes gst and polA were used as endogenous controls.

\section{$\boldsymbol{\beta}$-Galactosidase Assays}

Bacteria carrying lac $Z$ reporter vectors grown in appropriate media were harvested when bacterial growth reached exponential growth phase. Ortho-nitrophenyl- $\beta$-D-galactopyranoside (ONPG, Sigma, USA) was used as the substrate to measure $\beta$-Galactosidase activities and the Miller Units was calculated as described [7]. Each experiment was repeated 3 times, and the results were statistically analyzed using the student's $t$ test.
Construction of Tightly-Controlled FitR Expression Plasmid

To control expression of fitR, a fitR expression plasmid, pBAD-fitR1, was constructed by cloning the fitR gene into the expression vector pBAD22 [8]. Primers (Table 2) were used to PCR amplify fitR using high-fidelity DNA $p f u$ polymerase (Stratagene, USA). The forward primer contained an EcoR I site, and the reverse primer contained a Hind III site. The fragment was cloned into pBAD22 cleaved with EcoR I and Hind III. Protein expression from pBAD-fitR1 was tightly controlled by the arabinose pBAD promoter and was induced by addition of arabinose to the growth medium.

\section{Cloning and Purification of FitR}

A his-tagged fitR expression plasmid was constructed by cloning fitR into the expression vector pBAD22. A $6 \times$ His tag sequence was included in the reverse primer, which produced a C-terminal $6 \mathrm{X}$ His tag for protein purification (listed in supplemental materials Table 2). Then the amplified fragment was digested with EcoR I and Hind III, and 
Table 2. Oligonucleotide Primers Used in this Study

\begin{tabular}{|c|c|c|c|}
\hline & Gene & & Primer, 5'-3' \\
\hline \multicolumn{4}{|c|}{ PCR and cloning } \\
\hline & fitRl & forward & CGGAATTCGCGAGTCAGTTAACGCCT \\
\hline & & reverse & TATAAGCTTGCCATTCTTTCTTCCCGT \\
\hline & fitR2 & forward & CGGAATTCATGATCCAGAAAGATAAAGTGCG \\
\hline & fit promoter & forward & GTGCCACGTGTACGCCAACTTAAT \\
\hline & & reverse & TCGCGGATTGTCGCGAGTGAAA \\
\hline \multicolumn{4}{|l|}{ qPCR } \\
\hline & $f i t B$ & forward & GCACCATTGCACGTATCTTG \\
\hline & & reverse & ATAACGCTTTCGTTGGTTGC \\
\hline & fitC & forward & ACCTTGCACCCTCCCTTAAC \\
\hline & & reverse & CAAGCAGGGTAAATGTTCGC \\
\hline & fitD & forward & TCTGCTTGGTCATCAGGTTG \\
\hline & & reverse & AACAACGCCAGACGAGAACT \\
\hline & fitE & forward & TATCGGTGATTCAGGCAAACCAGG \\
\hline & & reverse & GTAATACCCGTCCCAGCGAATGAT \\
\hline & & reverse & AGCGCAGAACCGTAAACAGATAGG \\
\hline & pola & forward & TGAGTTCAAACGCTGGACTG \\
\hline & & reverse & TCTGCAACACTGGTTTCCTG \\
\hline
\end{tabular}

Restriction enzyme sites were depicted as underlined. In the reverse primer for $f i t R 2$, the $6 \times$ his tag sequence was indicated in bold.

ligated into plasmid pBAD22. Positive clones were identified through PCR and sequence analysis. In order to express the recombinant Fur protein, DH5 $\alpha$ cells carrying the positive fitR expression plasmid pBAD-fitR2 were grown in LB media. When bacterial growth reached an $\mathrm{A}_{600}$ of 0.6 , arabinose was added to the culture at a final concentration of $10 \mathrm{mM}$ to induce protein expression. After 5 hours of induction, cells were harvested by centrifugation $(4000 \mathrm{~g}$ for $20 \mathrm{~min}$ at $4^{\circ} \mathrm{C}$ ). Protein was purified by Ni-nitrilotriacetic acid (NTA) affinity chromatography, using Ni-NTA spin columns (Qiagen, USA), under denaturing conditions according to the manufacturer's instructions. Then the purified FitR was refolded by dialysis against $10 \mathrm{mM}$ Tris $(\mathrm{pH} 7.0)$. After the pellet was removed by centrifugation at $10,000 \mathrm{rpm}$ for 10min, supernatant was collected, analyzed by SDS- polyacrylamide gel electrophoresis, quantified using the Bradford assay kit (Bio-Rad, USA) and used as active FitR in the following assays.

\section{Electrophoretic Mobility-Shift Assay}

The electrophoretic mobility-shift assay was performed as follows. The $347 \mathrm{bp}$ intergenic region between fitA and $f i t B$, including the fit promoter, was PCR amplified using $p f u$ polymerase (Stratagene, USA) with synthesized primers (listed in Table 2). The purified DNA was end-labeled with digoxigenin using recombinant terminal transferase (Roche Applied Science, USA). Three nanograms of labeled DNA and various amounts of purified FitR protein were mixed and incubated at $37^{\circ} \mathrm{C}$ for $20 \mathrm{~min}$. The standard binding buffer was: $20 \mathrm{mM}$ Bis-Tris ( $\mathrm{pH} 7.0), 2.5 \mu \mathrm{g} / \mathrm{ml}$ sonicated salmon 

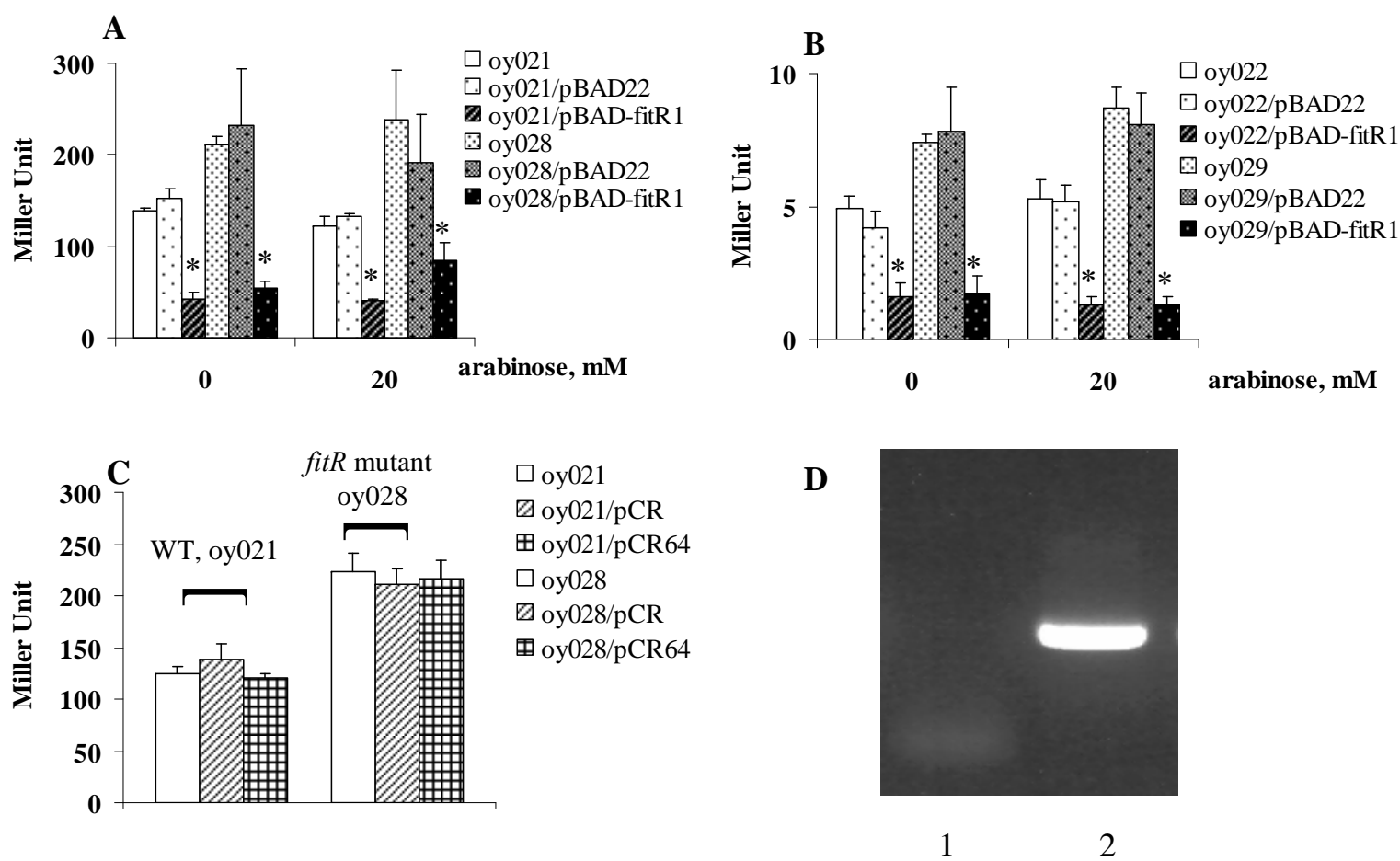

Fig. (1). FitR repressed fit expression, measured by lacZ reporter assays. Strains carrying $p M P$-fitA (A) or $p M P$-fitB (B) were grown in LB. When bacterial growth reached an A600 of 0.5-1.0, cells were harvested and $\beta$-galactosidase activities were measured as described (13). Y axis represents LacZ level, indicated in Miller Units. Star $\left(^{*}\right)$ represents statistically significant $(\mathrm{p}<0.05)$.

sperm DNA, $50 \mu \mathrm{g} / \mathrm{ml}$ poly $[\mathrm{d}(\mathrm{I}-\mathrm{C})], \quad 5 \%(\mathrm{w} / \mathrm{v})$ glycerol, $100 \mu \mathrm{g} / \mathrm{ml} \mathrm{BSA}, 1 \mathrm{mM} \mathrm{MgCl} 2,40 \mathrm{mM} \mathrm{KCl}$ and $100 \mu \mathrm{M} \mathrm{Mn}^{2+}$. Protein-DNA complexes were separated from unbound DNA on $5 \%$ nondenaturing polyacrylamide gels run at $50 \mathrm{~V}$ for 2 to $3 \mathrm{~h}$. The DNA was transferred onto a positively charged Nylon membrane (Roche Applied Science, USA) by electroblotting. The digoxigenin-labeled probes were subsequently detected by an enzyme immunoassay using an antibody (anti-digoxigenin-AP, Fab fragments) and the chemiluminescent substrate disodium 3-(4-methoxyspiro \{1,2dioxetane-3,2'-(5'-chloro)tricyclo[3.3.1.1 $\left.{ }^{3,7}\right]$ decan $\left.\}-4-y 1\right)$ phenyl phosphate (CSPD) (Roche Applied Science, USA).

\section{RESULTS AND DISCUSSION}

\section{FitR Represses fit Expression}

A lacZ reporter assay was employed to determine if FitR regulated fit expression. The intergenic DNA containing the bidirectional fit promoter, located between fitA and fitB, was cloned in two directions into the lacZ reporter vector, pMP220. The two fit-lacZ reporter plasmids were named pMP-fitA and pMP-fitB, and were used to measure the expression of fitA and fitB, respectively [2]. $\beta$-galactosidase activities were measured from exponential growth phase cells as described [7]. As shown in Fig. (1), the expression of fitB (Fig. 1A) in strains containing pMP-fitB was very similar to the strain carrying $\mathrm{pMP}-$ fitB and the cloning vector pBAD22. The wild type strain (oy021) containing the plasmid pMP-fitB expressed 139 units of $\beta$-galactosidase (Fig. 1A). When FitR was expressed from pBAD-fitR1 in wild type strains, this strain expressed 43 units of $\beta$-galactosidase, which showed that expression of fitB was repressed 3.2 fold. Further, cells expressed 211 units of $\beta$-galactosidase in the
fitR knockout mutant, indicating that $f i t B$ was de-repressed 4.9 fold in the fitR mutant (oy028). Finally, when the fitR mutation was complemented in trans- by pBAD-fitR1, cells expressed 55 units of $\beta$-galactosidase, which confirmed the observation that fit was repressed by FitR. A similar regulation pattern was obtained for strains containing pMP-fitA (Fig. 1B). These data demonstrated that FitR does repress fit expression.

The repression of fit by FitR was also found even when FitR expression was not induced ( $0 \mathrm{mM}$ arabinose, Fig. 1), which might be the result of leaky expression of fit $R$ from pBAD-fitR1. To test this, we cloned fitR into the plasmid pCR-XL-TOPO, a cloning vector without a ribosome binding site (RBS). The new plasmid was designated pCR64. When this plasmid was introduced into wild type strains oy021 or fitR mutant oy028, strains oy021/pCR-XL-TOPO, oy021/pCR64, oy028/pCR-XL-TOPO and oy028/pCR64 expressed 129, 120, 228 and 217 units of $\beta$-galactosidase, respectively, which demonstrated that fit expression was not repressed without fitR expression (Fig. 1C). Further, we examined fitR transcription using RT-PCR. As shown in Fig. (1D), the fitR transcript was detected in cells carrying pBAD-fitR1 grown under non-induced condition (lane 2). Thus we concluded that the repression of fit by pBAD-fitR 1 when cells were grown in LB containing $0 \mathrm{mM}$ arabinose resulted from the leaky expression of FitR from $\mathrm{pBAD}$ promoter. The fact that pBAD22 is quite leaky has been found in other studies $[8,9]$. The regulation of the fit system by FitR was also examined using a quantitative RT-PCR analysis. Compared to gene expression in wild type strain $E$. coli $\mathrm{i} 484$, fitB was repressed 1.8 fold when FitR was expressed from $\mathrm{pBAD}$-fitR1 and de-repressed 3.2 fold when fitR was mutated. In addition, when the fitR mutation was comple- 

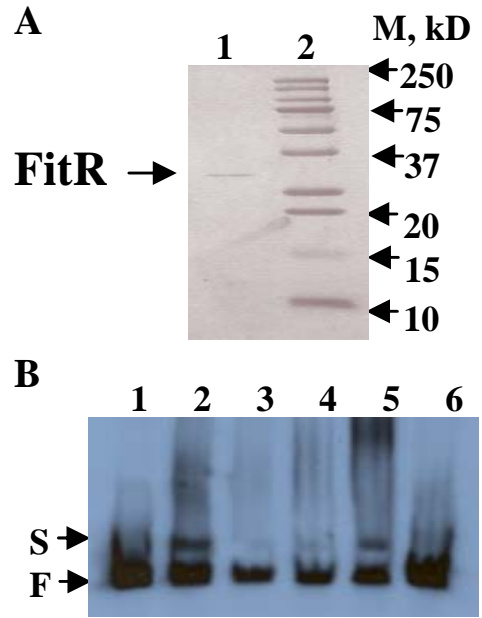

Fig. (2). A), Purified recombinant His-tagged FitR protein analyzed by a 15\% SDS-PAGE gel. Lane1, His-tagged FitR; lane 2, standard protein Marker. B), FitR binds to the fit promoter. All the lanes contained 3ng end-labeled fit promoter DNA. Lane 1 contained no protein; lanes 2-5 contained 100, 10, 20, and 50ng purified FitR, respectively. Lane 6 contained 50ng FitR and 150ng unlabeled fit promoter DNA. S and F indicate FitR bound and free DNA bands, respectively.

mented by pBAD-fitR1, fitB was repressed 6.8 fold compared with gene expression levels in the fitR mutant. Similar results were obtained for fitA expression. These results demonstrated that FitR represses the expression of the fit system.

\section{Binding of Recombinant FitR to the fit Promoter}

If FitR directly regulates the fit system, it would be expected to bind to the fit promotor. To obtain protein FitR, a fitR over-expression plasmid, pBAD-fitR2, was constructed by cloning fitR into plasmid pBAD22. E. coli $\mathrm{DH} 5 \alpha$ cells carrying pBAD-fitR2 were grown in LB and protein expression was induced by $10 \mathrm{mM}$ arabinose. As shown in Fig. (2A), the purified recombinant FitR was found to have a molecular weight of $28 \mathrm{kDa}$ (lane 1 ), which is consistent with the predicted molecular weight based on sequence analysis. To determine if FitR bound to the fit promoter, an electrophoretic mobility-shift assay (EMSA) was performed. As shown in Fig. (2B), the purified FitR was found to bind to the fit promoter DNA when used at 20,50, or 100ng (lane 2, $3,4,5)$. When a 50 fold excess of non-labeled fit promoter DNA was used as a specific competitor in the reaction, the binding of FitR was found to be inhibited (lane 6). These results suggested that FitR binds specifically to the fit promoter.

\section{FitR Repression of the fit System was Independent of Fur's Regulation}

The $E$. coli fit system has been found to be repressed by Fur-Fe ${ }^{2+}$ complex (Ouyang and Isaacson unpublished). Therefore, fit is regulated by two repressors: Fur and FitR. Here we investigated the possibility that these two repressors regulated fit expression independently. Initially, a lacZ reporter assay was performed to determine if FitR needed iron as a cofactor to repress fit expression. As shown in Fig. (3A), the wild type strain containing pMP-fitB (oy021) expressed 168 and 525 units of $\beta$-galactosidase under iron-rich and
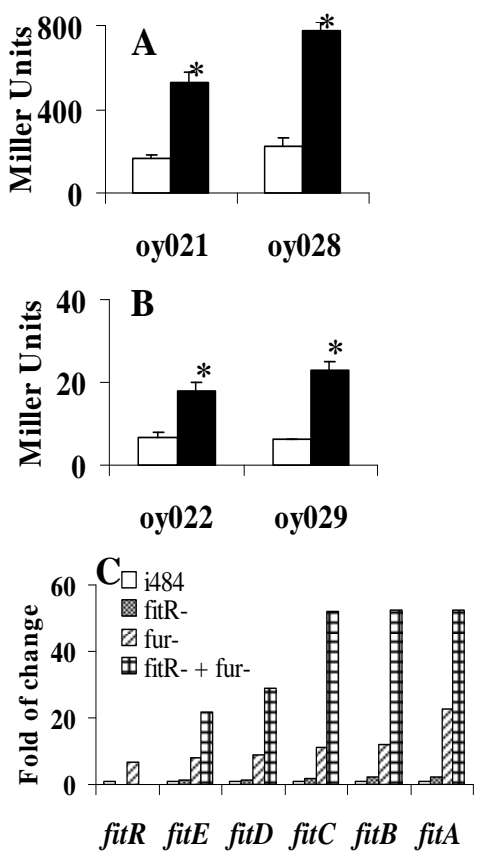

Fig. (3). Fur and FitR repressed fit expression independently. A, B), Iron is not required for repression of fit expression by FitR. E. coli strains carrying pMP-fitB (A) or pMP-fitA (B) were grown in LB (open bar) or LB with $100 \mu \mathrm{M} 2$, 2'-dipyridyl (filled bar). Star (*) represents statistical significant $(\mathrm{p}<0.05)$. C), Gene expression levels measured using quantitative RT-PCR analysis. RNA was isolated from bacteria grown in LB for 3 hours. Strains used were: E. coli i484; oy071 (i484, fur::DHFR-1), oy077 (fitR::kan-2), and oy078 (fur and fitR double mutant). Results were shown as fold of change: ratio of gene expression levels under other conditions to the expression level in $E$. coli i484 (open bar). Values are the means from three independent experiments.

iron-deficient conditions, respectively. In the fitR mutant (oy028), cells expressed 227 and 777 units of $\beta$-galactosidase under iron-rich and iron-deficient conditions, respectively. Similar results were obtained for pMP-fitA (Fig. 3B). These results indicated that fit expression was still induced by iron deprivation when fitR was mutated, which in turn suggested that iron and FitR separately repressed fit expression. In addition, a quantitative RT-PCR analysis was employed to study if the repression of FitR is independent of expression of Fur. As shown in Fig. (3C), fitA expression was induced 23 fold in the fur mutant, 2.2 fold in the fitR mutant, and 52 fold in the fitR and fur double mutant. These data suggested that FitR and Fur repressed fitA independently. Similar results were observed for other fit genes.

In $E$. coli, for those well-studied iron transporters, such as $f e p, f h u, f e c, f e o$, they are all regulated by the global regulator, Fur (ferric uptake regulator), through binding of Fur$\mathrm{Fe}^{2+}$ complex to the Fur box in the gene promoters [10, 11, $12]$. And except for the fec system, most E. coli iron transporters do not have their own regulators [13, 14, 15, 16]. Interestingly, the $E$. coli fit system is regulated by two independent repressors, an auto-regulator FitR and a global regulator Fur. This elegant regulation may indicate the important role of fit in E. coli metabolism. So far we don't know if FitR is a global regulator or if any other factor is involved in the regulation of the fit system. Further studies, for example, identification of the exact binding site of FitR or Fur on fit 
promoter, whether there is a synergy in binding by addition of Fur and FitR, and whether other proteins might be involved in the expression of fit, etc, may elucidate the detailed regulation mechanism of this novel iron transporter.

\section{CONCLUSION}

In conclusion, an auto-repressor, FitR, was identified for the $E$. coli fit iron transport system in this report.

\section{ACKNOWLEDGEMENTS}

We thank Dr. Sandra K. Armstrong, University of Minnesota, for providing the plasmid pMP220. This work was supported in part by a grant from the USDA-NRI 200235201-12542.

\section{REFERENCES}

[1] Griffiths E. Iron in biological systems. In Bullen J, Griffiths E, Eds., Iron and infection. Wiley, Chichester, United Kingdom. 1999; $1-26$

[2] Ouyang Z, Isaacson R. Identification and characterization of a novel ABC iron transport system, fit, in Escherichia coli. Infect Immun 2006; 74: 6949-56.

[3] Bateman A. The SIS domain: a phosphosugar-binding domain. Trends Biochem Sci 1999; 24: 94-95.

[4] Poulsen T S, Chang YY, Hove-Jensen B. D-Allose Catabolism of Escherichia coli: Involvement of alsI and Regulation of als Regulon Expression by Allose and Ribose. J Bacteriol 1999; 181: 712630.

[5] Datsenko KA, Wanner BL. One-step inactivation of chromosomal genes in Escherichia coli K-12 using PCR products. Proc Natl Acad Sci USA 2000; 97: 6640-45.
[6] Goryshin IY, Reznikoff WS. Tn5 in vitro transposition. J Biol Chem 1998; 273: 7367-74.

[7] Miller JH. Experiments in Molecular Genetics. Cold Spring Harbor laboratory Press, Cold Spring Harbor, NY. 1972.

[8] Guzman LM, Belin D, Carson M. J. Beckwith J. Tight regulation, modulation, and high-level expression by vectors containing the arabinose PBAD promoter. J Bacteriol 1995; 177: 4121-30.

[9] Wei Y, Newman, E.B. Studies on the role of the $m e t K$ gene product of Escherichia coli K-12. Mol Microbiol 2002; 43:1651-56.

[10] Crosa J.H. Signal transduction and transcriptional and posttranscriptional control of iron-regulated genes in bacteria. Microbiol Mol Biol Rev 1997; 61: 319-36.

[11] Faraldo-Gomez JD, Sansom MS. Acquisition of siderophores in gram-negative bacteria. Nat Rev Mol Cell Biol. 2003; 4: 105-116.

[12] Hantke, K. Iron and metal regulation in bacteria. Curr Opin Microbiol 2001; 4: 172-177.

[13] Braun V., M. Braun. Iron transport and signaling in Escherichia coli. FEBS Lett 2002; 529: 78-85.

[14] Braun V, Mahren S, Ogierman M. Regulation of the FecI-type ECF sigma factor by transmembrane signalling. Curr Opin Microbiol 2003; 6:173-180.

[15] Enz S, Mahren S, Stroeher UH, Braun V. Surface signalling in ferric citrate transport gene induction: interaction of the FecA, FecR, and FecI regulatory proteins. J Bacteriol 2000; 182: 637-646.

[16] Ochs M, Veitinger S, Kim I, Welz D, Angerer A, Braun V. Regulation of citrate-dependent iron transport of Escherichia coli: FecR is required for transcription activation by FecI. Mol Microbiol 1995; 15: 119-132.

[17] Khan MA, Isaacson RE. Identification of Escherichia coli genes that are specifically expressed in a murine model of septicemic infection. Infect Immun 2002; 70: 3404-12.

[18] Sambrook J, Russell DW. Molecular cloning. A laboratory manual. 3rd ed. Cold Spring Harbor Laboratory Press: Cold Spring Harbor, NY 2001.

[19] Spaniak HP, Okker RJH, Wijffelman CA, Pees E, Lugtenberg BJJ. Promoters in the nodulation region of the Rhizobium leguminosarum Sym plasmid pRL1JI. Plant Mol Biol 1987; 9: 27-39.

(C) Ouyang and Isaacson; Licensee Bentham Open.

This is an open access article distributed under the terms of the Creative Commons Attribution License (http://creativecommons.org/licenses/by/2.5/), which permits unrestrictive use, distribution, and reproduction in any medium, provided the original work is properly cited. 